\title{
Overview of Carrier frequency concealment Radar Signal Design
}

\author{
Guanyi Chen ${ }^{a, *}$, Limin Liu \\ Department of Electronic and Optics Engineering, Ordnance Engineering University, Shijiazhuang, \\ China. \\ achenguanyiyi@qq.com
}

\begin{abstract}
Keywords: Radar Signal Design, Carrier frequency concealment Radar Signal, Composite signal design.
\end{abstract}

\begin{abstract}
In the increasingly fierce modern electronic warfare, it is crucial to hide the relevant parameters of the radar signal itself. In this paper, the background and significance of radar signal design are described. The development and current status of radar signal design are studied. The design status of carrier frequency concealment radar signal is analyzed. Carrier frequency concealment radar signal design research is still in its infancy, the study of a variety of combined signals help to design a better carrier concealment signal.
\end{abstract}

\section{Introduction}

In the modern military confrontation, the radar as the main representative of the various active detection equipment is an important means to obtain enemy target information. In modern warfare, the effective confrontation of radar can provide a strong guarantee for the elimination of the enemy, the fulfillment of the task and the protection of the enemy. Radar confrontation as well as the important means and important guarantee for obtaining the information superiority and military superiority plays a more and more important role. However, with the rapid development of electronic confrontation and electronic anti-confrontation, especially in the past 20 years, the various radiation sources and the formation of electromagnetic waves are extremely congested space domain, time domain and frequency domain. In such a complex electromagnetic environment, how to design anti-jamming radar signals which can effectively conceal the radar signal parameters ${ }^{[1]}$ is an urgent issue to be discussed in modern electronic warfare.

Radar has played an increasingly important role in modern military confrontation Since World War II. Electronic detection equipment and military high-tech have largely determined the success or failure of war, and they have also been unprecedentedly developed and applied in the military field. The confrontation based on radar is becoming increasingly fierce. The United States and the United Kingdom deployed in German theater dozens and the important technical indicators of radars in advance before Normandy Landing. Thus the enemy radar system was almost paralyzed while the allied forces controlled their loss rate of coalition ship within $0.3 \%$. In the Gulf War, the forces of many countries that were headed by the United States carried out electronic reconnaissance and electronic interference on a large scale in the battlefield resulting the enemy radars almost ineffective.The US military deployed thousands of stealth fighter Military operations which cost almost no loss. Throughout the modern military war between World War II, it is easy to see that the party that can take the initiative and dominance in the radar confrontation will eventually win the war. Electronic confrontation and counter-resistance, especially the radar as the representative of the confrontation has become a modern war victory or not the primary factor. It is of great and far-reaching significance for a country's national defense to effectively hide its own radar signal parameters and improve the anti-jamming performance of the signal under complex electromagnetic environment.

The radar would be disturbed if it was detected first. Radar reconnaissance is based on the principle of intercepting, measuring, analyzing, identifying and locating the radiated electromagnetic signals of radars to obtain the carrier frequency, signal waveform, pulse width, pulse repetition frequency, signal intensity, polarization mode, beam width, Scanning mode, radar deployment, functions and other 
major tactical technical parameters with electronic reconnaissance equipment. The early radar anti-reconnaissance equipment measures only the carrier frequency of the enemy radar signal, combined with the arrival direction of the radar signal measured by the directional antenna, and guides the interfering transmitting system in frequency and direction. Reconnaissance receivers achieves intelligence reconnaissance, radar search, alarm and other tasks ${ }^{[2]}$ mainly through the radar signal capture and analysis. Modern radar system reconnaissance equipment can realize more direct and indirect derivation of radar signal characteristics, but the carrier frequency of radar signal is still one of the most important characteristic parameters that need to be measured.

\section{Situation of radar signal design}

With the rapid development of military science and technology, the application and living environment of modern radars are threatened by the four major threats: "electronic jamming", "stealthy targets", "anti-radiation missiles" and 'low-altitude / ultra-low penetration" ${ }^{\text {'3] }}$. The radar obtains target's reflection of electromagnetic waves by transmitting radar signals, correlates the target echoes and obtains the target information, so as to realize the functions of radar target detection, tracking and identification. Radar transmission signals not only determine the resolution, measurement accuracy and interference suppression capabilities and other properties of the radar system, but also determine the subsequent signal processing methods. Therefore, in order to improve the performance characteristics of the radar system and the capability of "four resistance", the research on the design, optimization and selection of radar signals has very important strategic and application significance ${ }^{[4]}$.

Radar signal theory includes two aspects: radar signal processing theory and radar signal design theory. The basis of radar signal design is the theory of radar signal processing which is developed on the theory of signal detection and fuzzy theory ${ }^{[5,6]}$. At the same time, radar signal design promotes the development of radar signal processing theory in turn. Radar signal processing theory began in the 1940s, represented by the matched filter theory proposed by North in 1943 . The matched filter theory made the output SNR (signal to noise ratio) reach the maximum under the Gaussian white noise condition, which greatly improves the radar detection performance. Urkowitz extended the theory of matched filters to the application of colored noise and proposed the whitening filter and inverse filter theory. Subsequently, Manasse proposed the best filter for the coexistence of white noise and clutter interference, which provided a theoretical basis for the suppression of clutter radar signal optimization problems. In 1953, Woodward proposed a well-known radar fuzzy theory that included new concepts such as fuzzy functions and resolving constants. This theory not only provided the theoretical basis of the radar signal resolution, but also involved the radar signal design problem for the first time. Under the premise of using the optimal radar signal processing, the correlation between the radar signal transmitted by the radar system, the resolution, the ambiguity, the measurement accuracy and the clutter suppression capability of the radar system was researched, which greatly facilitated the radar Signal design in-depth exploration and research. The subsequent radar signal design has been studied along two different approaches based on this theory. One method was the waveform synthesis method proposed by Sussman et al. In the 1960s, and the optimal radar signal was obtained through the optimal synthesis method of fuzzy functions. However, the method was computationally intensive, and the resulting complex modulated radar signal was technically difficult to implement. And the other is the "radar signal selection method" which takes into account the ease of realization of the technology and selects the appropriate signal form and waveform parameters, proposed by Rihaczek in 1971 based on the principle of matching the target environment map and radar signal blur graph. By selecting the appropriate signal forms and parameters, the method took into account the project to achieve and made the system performance indicators meet the requirements. Thus it has been widely used in engineering practice. Typical pulse radar could not take into account the system detection performance, distance and speed measurement accuracy and resolution simultaneously. In order to solve this problem, a variety of complex waveforms of radar signals ${ }^{[7]}$ have been carried out. The earliest practical application is the linear FM signal. After that, nonlinear time-frequency modulation signal, phase 
encoding signal, coherent pulse train signal and random time-bandwidth radar signals have been proposed one after another.

\section{Present situation of radar carrier frequency concealment signal design}

Radio frequency cover is a classic radar anti-jamming measures, which was first used in anti-noise aiming interference. Radio frequency cover signals are designed to protect the real working frequency of the radar with a fraudulent radio frequency pulse signal waveform ${ }^{[8]}$. The radar radio frequency covered measures come from the accidental discovery in the engineering practice of the radar transmitter, that is, the engineering effect of the pulse traveling wave tube. When the radar signal is transmitted, pulse TWT (traveling wave tube), send the RF pulse signal into the RF input radio frequency input interface. The traveling wave tube should be started by the video pulse of the video input interface, the video pulse is strictly synchronized with the RF input pulse in theory. But in fact, if the synchronization is not good, Parasitic modulation frequencies will be generated at the radar transmitter front and back edges. Besides, the modulation frequency may be different from the main frequency of the radar. This type of waveform may allow the frequency-measuring receiver sampling the leading edge of the pulse to measure only the parasitic modulation frequency, so that the parasitic modulation frequency will cover the actual radar operating frequency and may mask the frequency of the real signal. However, with the development of broadband interference technology, the signal of radio frequency protection gradually faded out of people's view. At this stage, many scholars turned to low-cut-off radar research.

In current radar anti-reconnaissance waveform design, there is no radar emission waveform specifically designed for radar carrier frequency concealment, but LPI(low probability of interception) radar waveform design can hide some parameters of radar signal. Because of the simple form of phase coding and LFM (linear frequency modulation) signal, it is easy to implement in engineering. So these two kinds of signal forms are used in most of the existing low intercept radars. In addition, typical LPI radar signals include frequency-coded signals and compound modulated signals ${ }^{[9]}$. Although each signal has good low probability of interception performance, there are always some shortfalls in the signal itself. The existing technical conditions are difficult to achieve a large time-bandwidth signal, which also limits the low signal acquisition performance ${ }^{[10]}$.

Radar signal design is divided into single signal design and combined signal design, and the above mentioned early radio frequency covered signal is a combination of signal. Study of radar signals a trend in the 20th century is the use of appropriate combinations of existing radar signals, developed a new signal in the form of performance to meet the technical requirements in order to obtain more excellent radar signal waveform. The advantage of the combined signal is that it can use all the achievements of the existing radar technology, but also conducive to the transformation of active radar.

Therefore, in order to be able to avoid weaknesses of single signal, the combined modulation signal generated by the corresponding modulation was born. Composite modulation signal refers to two or more than two modulation methods to modulate the signal of the new signal. Compound modulation signal is a new signal that is modulated with two or more than two modulation modes. The combined modulation signal can combine the advantages of the modulation method of a single signal to obtain better distance and speed resolution, reduce the probability that the signal is intercepted, identified and tracked by the non-cooperative receiver, and better hide the relevant parameters of its own signal. In addition, in order to increase the difficulty of being intercepted, some complex LPI signal forms similar to complex modulation have also been gradually applied to modern radar systems, such as chirp signals and multiphase coded signals ${ }^{[11]}$ derived from chirp. The main idea of combinational signal design is studied from the aspects of frequency relationship design, phase relation design, power relationship design, frequency modulation mode design and detection distance analysis. 


\section{Summary}

Some of the radar signals are designed to improve the radar's stealth performance, such as reducing the transmit power of the signal. Some of the radar signals are designed to improve the radar's detection performance, such as resolution and ranging accuracy. Based on the carrier frequency concealment of the radar signal design is mainly for the instantaneous frequency measurement system of the receiver design. The current composite signal can be combined with the advantages of each signal in the combined signal to obtain further detection range, higher resolution, more subtle signal parameters, such as carrier frequency, modulation and so on. So the current stage of the carrier frequency concealment signal design should be put into the combined signal design. As long as the in-depth study, the composite modulation form of the combined signal carrier frequency concealment performance and anti-jamming performance have more room for development.

\section{References}

[1] Zhang, X. X. 2003. Development of lpi radar. Modern Radar, 25(12):1-4.

[2] He, P. January 2016. Theory of Radar Countermeasure. National Defense Industry Press.

[3] Yang, Y. S. 2014. Research on the LPI radar signal examination system design. Nanjing University of Aeronautics and Astronautics.

[4] Tong, J. 2008. Research on the lpi radar signal examination system design. Foreign Electronic Measurement Technology.

[5] Jiu, B., Liu, H. W., Liu, Z., \& Wu, S. J. 2012. Sequential waveforms optimization for wideband target recognition radar based on kernel method. IEEE Cie International Conference on Radar (Vol.2, pp.1267-1271). IEEE.

[6] Bo, J., Liu, H., Chen, B., \& Liu, Z. 2013. Waveform design for wideband radar target recognition based on eigensubspace projection. Iet Radar Sonar \& Navigation, 7(6), 702-709.

[7] Levanon, N., and Mozeson, E. 2004. Radar Signals. IEEE Xplore.

[8] Zhou, W., Wang, P., Zhang, J., \& Tong, L. 2013. Analysis and countermeasures of radar radio frequency-screen signal. Aerospace Electronic Warfare.

[9] Cao, S. Q. 2013. Active Phased Array Radar Signal Design And LPI Performance Research. University of Electronic Science and Technology of China.

[10]Sun, Z. Y., Jiang, Q. X., Cui-Qiong, M. O., and Liang-Hao, X. U. 2015. A design of counter reconnaissance based on subsection lfm modulation random waveform. Radar Science \& Technology.

[11]Peng, Y., Yang, J., Fang, Y. U., and Duan, Y. 2015. Orthogonal poly-phase coded signals optimization for mimo radar. Com-puter Engineering and Applications. 